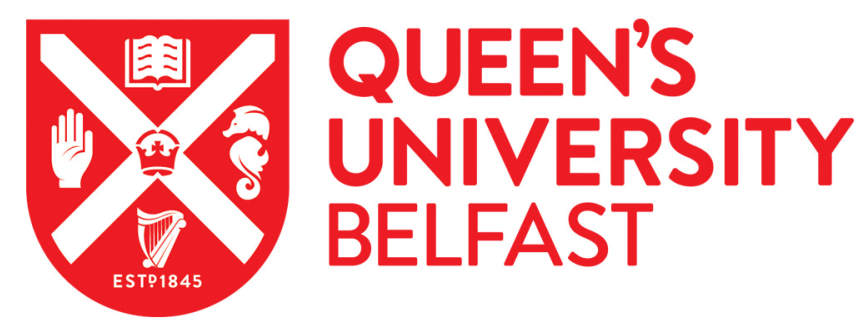

\title{
Absolute calibration of microchannel plate detector for carbon ions up to $250 \mathrm{MeV}$
}

Mcllvenny, A., Doria, D., Romagnani, L., Ahmed, H., Martin, P., Williamson, S. D. R., Ditter, E. J., Ettlinger, O. C., Hicks, G., Mckenna, P., Najmudin , Z., Neely, D., Kar, S., \& Borghesi, M. (2019). Absolute calibration of microchannel plate detector for carbon ions up to $250 \mathrm{MeV}$. Journal of Instrumentation, 14, [C04002]. https://doi.org/10.1088/1748-0221/14/04/C04002

Published in:

Journal of Instrumentation

Document Version:

Peer reviewed version

Queen's University Belfast - Research Portal:

Link to publication record in Queen's University Belfast Research Portal

Publisher rights

Copyright 2019 IOP. This work is made available online in accordance with the publisher's policies. Please refer to any applicable terms of use of the publisher.

\section{General rights}

Copyright for the publications made accessible via the Queen's University Belfast Research Portal is retained by the author(s) and / or other copyright owners and it is a condition of accessing these publications that users recognise and abide by the legal requirements associated with these rights.

Take down policy

The Research Portal is Queen's institutional repository that provides access to Queen's research output. Every effort has been made to ensure that content in the Research Portal does not infringe any person's rights, or applicable UK laws. If you discover content in the Research Portal that you believe breaches copyright or violates any law, please contact openaccess@qub.ac.uk. 


\title{
Absolute calibration of microchannel plate detector for carbon ions up to $250 \mathrm{MeV}$
}

\author{
A. Mcllvenny ${ }^{a}$ D. Doria, ${ }^{b}$ L. Romagnani, ${ }^{c}$ H. Ahmed, ${ }^{a}$ P. Martin, ${ }^{a}$ S. D. R. WIIliamson, ${ }^{d}$ E. J. \\ Ditter, ${ }^{e}$ O. Ettlinger, ${ }^{e}$ G. S. Hicks, ${ }^{e}$ P. McKenna, ${ }^{d}$ Z. Najmudin, ${ }^{e}$ D. Neely, ${ }^{f}$ S. Kar,${ }^{a}$ and M. \\ Borghesi $^{a, 1}$ \\ ${ }^{a}$ Centre for Plasma Physics, Queen's University Belfast, University Road BT71NN, United Kingdom \\ ${ }^{b}$ Extreme Light Infrastructure - Nuclear Physics (ELI-NP), Horia Hulubei Institute for Nuclear Physics \\ (IFIN-HH) \\ ${ }^{c}$ LULI, École Polytechnique, CNRS, Route de Saclay, 91128 Palaiseau Cedex, France \\ ${ }^{d}$ SUPA, Department of Physics, University of Strathclyde, Glasgow G4 ONG, United Kingdom \\ ${ }^{e}$ The John Adam's Institute, Imperial College London, London, SW72AZ, United Kingdom \\ ${ }^{f}$ Central Laser Facility, Rutherford Appleton Laboratory, Oxfordshire OX11 OQX, United Kingdom \\ E-mail: m.borghesi@qub.ac.uk
}

\begin{abstract}
A 375TW 40fs pulse was used at the ASTRA GEMINI facility located at the Rutherford Appleton Laboratory U.K. for investigating novel ion acceleration regimes employing ultrathin foil targets. An online detection system consisting of a Thomson Parabola Spectrometer (TPS) Microchannel Plate (MCP) was used to determine maximum energies and spectra per species. The response of the MCP was calibrated for absolute particle (carbon) number per steradian using CR-39 up to $21 \mathrm{MeV} /$ nucleon. This calibration provides a useful reference for a widely used diagnostic arrangement.
\end{abstract}

Keywords: Lasers, Particle detectors, Ion sources (positive ions, negative ions, electron cyclotron resonance (ECR), electron beam (EBIS)), Spectrometers, Plasma diagnostics - charged-particle spectroscopy

\footnotetext{
${ }^{1}$ Corresponding author.
} 


\section{Contents}

1 Introduction 1

2 Experiment 2

2.1 Experimental Set-up 2

2.2 MCP detector 3

2.3 Calibration 3

2.4 Spectra 5

3 Conclusions $\quad 5$

\section{Introduction}

Ion acceleration from high intensity $\left(>10^{18} \mathrm{~W} / \mathrm{cm}^{2}\right)$ lasers has been extensively explored using a variety of laser and target parameters as a means of generating high energy, short duration (ps) ion bursts. Most of the focus has been towards Target Normal Sheath Acceleration (TNSA) generating short pulses of 10s of MeV multi-species ions from the target bulk and rear contaminants[1]. This is typically done using targets with thickness of a few microns, where ions are accelerated by the strong sheath field (order of $\mathrm{TV} / \mathrm{m}$ ) created by the hot electrons at the target surfaces.

Theoretical and numerical investigations suggest that for the next generation of lasers with higher intensities $\left(>10^{22} \mathrm{~W} / \mathrm{cm}^{2}\right)$, radiation pressure acceleration (RPA) will begin to dominate, potentially achieving ion energies in the $100 \mathrm{~s}$ of $\mathrm{MeV}$ to $\mathrm{GeV}$ range[2]. Recent studies have shown that RPA can become the dominant acceleration mechanism already at current intensities by careful control of the target thickness and polarization of the laser[3]. Significant attention has been devoted recently to the development of laser driven ion accelerators as a compact source of high energy ions with clinical interest $[4,5]$ which motivates the effort in understanding and characterising these beams.

Laser-based ion acceleration experiments routinely utilize absolutely calibrated detectors such as radiochromic film (RCF), CR-39 (Columbia Resin 39, a solid-state track detector) and Image plates (IP), detailed in [6]. These are used either in a stack arrangement [7] or, in the case of IP or CR-39, in the detection plane of a Thomson Parabola Spectrometer (TPS). Calibrations for these detectors exist up to $10 \mathrm{~s}$ of $\mathrm{MeV}$ for protons and heavier ions [8-10]. The detectors are typically deployed within the evacuated interaction chamber and thus only after breaking vacuum and processing the detector, information on the energies achieved in a shot can be obtained. This delay can be removed with the use of online detectors such as MCPs, which provide a real-time response and can be operated without breaking the vacuum in the chamber.

We report here on the calibration of a MCP assembly up to $21 \mathrm{MeV} / \mathrm{u}(252 \mathrm{MeV})$ for $\mathrm{C}^{6+}$ under conditions of relevance to irradiation of ultrathin foil investigations similar to those reported in [3]. The calibration data was collected in a follow up experiment aiming to extend the previous dataset 


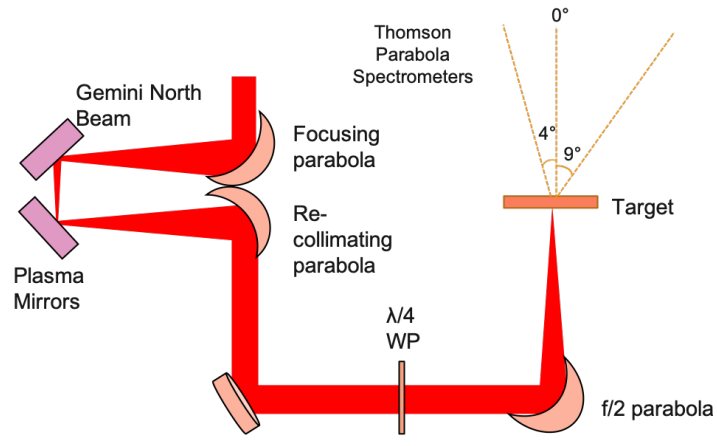

(a)

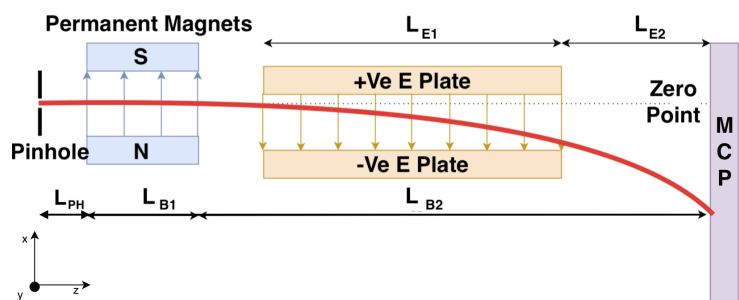

(b)

Figure 1: (a) Experimental set-up using double plasma mirror arrangement in the GEMINI target area (b) $0^{\circ}$ TPS Dimensions: $\mathrm{L}_{P H}=67 \mathrm{~mm}, \mathrm{~L}_{B 1}=50 \mathrm{~mm}, \mathrm{~L}_{B 2}=555 \mathrm{~mm}, \mathrm{~L}_{E 1}=200 \mathrm{~mm}, \mathrm{~L}_{E 2}=$ $335 \mathrm{~mm}$, Pinhole Size $=0.4 \mathrm{~mm}$, B Field Strength $=0.972 \mathrm{~T}$, E field Strength $=25 \mathrm{kV} / \mathrm{cm}$

and to advance our understanding of radiation pressure acceleration, and its dependence on target thickness and laser polarization.

\section{Experiment}

\subsection{Experimental Set-up}

The experiment was set up as shown in figure 1a, using a double plasma mirror configuration to produce a contrast $<10^{-12}$ on the nanosecond timescale in order to preserve the integrity of the foils; this reduces the amount of front surface expansion before the main pulse arrives providing a steep density gradient for the interaction. The beam passed through a quarter wave plate, used to alter the polarization between linear and circular. The beam was focused using the $f / 2$ parabola and incident at $0^{\circ}$ on target. This produced $\sim 6 \mathrm{~J}$ on target and an intensity of $\sim 5 \times 10^{20} \mathrm{~W} / \mathrm{cm}^{2}$. Amorphous carbon targets $2-100 \mathrm{~nm}$ thick were used. The diagnostic set-up was optimized for the detection of high energy carbon ions and protons, with a TPS, as expected from bulk acceleration in the ultra-thin target regime. To improve the number of shots that could be taken between chamber cycles (replacing targets and other detectors), MCPs were used as the detectors to record the particle spectra. A calibration for this detector was an objective for present and future use, as required in order to achieve quantitative information on the ion spectra.

Using the information in figure $1 \mathrm{~b}$ along with the equation 2.1 below, the vertical deflection (SB) can be associated to a given ion energy (where $r_{L}$ is the Larmor radius associated with the B field). The electric field separates the species and the deflection (SE) is given by equation (2.2).

$$
S_{B}=r_{L}-\sqrt{r_{L}^{2}-L_{B 1}^{2}}+\frac{L_{B 1} L_{B 2}}{\sqrt{r_{L}^{2}-L_{B 1}^{2}}}
$$

$$
S_{E}=\frac{q E L_{E 1}}{m v_{0}^{2}}\left[\frac{L_{E 1}}{2}+L_{E 2}\right] \frac{r_{L}^{2}}{\sqrt{r_{L}^{2}-L_{B 1}^{2}}}
$$




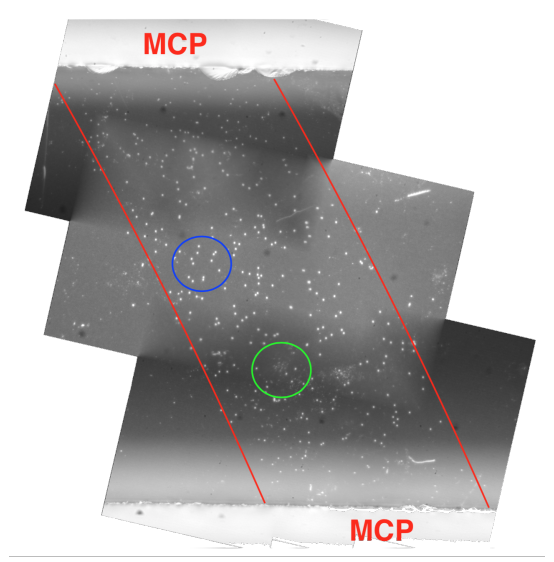

(a)

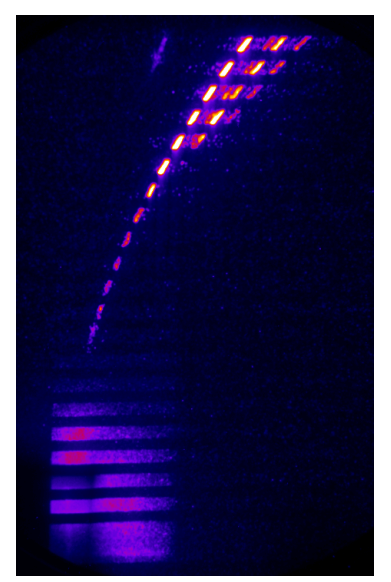

(b)

Figure 2: (a) Mosaic image of CR-39 at x10 magnification showing the width of the parabolic ion trace (red lines) and the CR-39 - MCP interface at 4.1MeV/u (top) and $4.4 \mathrm{MeV} / \mathrm{u}$ (bottom). The blue circle represents $\mathrm{C}^{6+}$ and green represents noise from damage particles (b) Image of MCP with slotted CR-39 placed in front of it forming regular interfaces between the two.

\subsection{MCP detector}

The calibration presented was performed using a Hamamatsu MCP F2226-14PF227 on the $0^{\circ}$ TPS. This is a $77 \mathrm{~mm}$ diameter circular MCP with a thickness of $1 \mathrm{~mm}$ and a $\mathrm{P} 47$ phosphor screen. The $\mathrm{MCP}$ acts as an electron multiplier to incident ionizing radiation where the electrons are accelerated by a potential difference towards the phosphorous screen. The fluorescence has a $10 \%$ decay time of less than $1 \mathrm{~ms}$ and this was captured with an Andor Neo CCD with a longer exposure (typically $100 \mathrm{~ms}$ ) using the same trigger as the laser. With this arrangement, the image can be obtained remotely and immediately after the shot and hence some basic information on the particle beam e.g. a measure of the maximum energy per species, can be obtained almost instantly. The space between the MCP screen and CCD was enclosed and light-tight to remove any background noise.

MCPs require a high vacuum $\left(<10^{-6} \mathrm{mbar}\right)$ to safely operate and a large potential difference $(3 \mathrm{kV})$ so that even a small number of incident particles can generate a sufficiently high signal on the phosphorus screen [11]. The response of the MCP to high energy carbon ions has not yet been well characterised, and calibration data exist only for protons and lower energy carbon ions [12,13]. The response is not necessarily expected to be linear against particle energy due to the large variance in the LET for the different energy ions produced in a single shot. In the context of our experimental program aimed at characterizing RPA, it is useful to extract an energy spectrum in absolute terms in order to identify features associated with the acceleration mechanism, particularly in relation to the carbon ions from the target bulk.

\subsection{Calibration}

In order to perform the calibration, slotted CR-39 (slot width of 1mm) was placed in front of the $\mathrm{MCP}$, on the $0^{\circ}$ line, on a shot (e.g. figure $2 \mathrm{~b}$ ). This calibration procedure, already reported in 


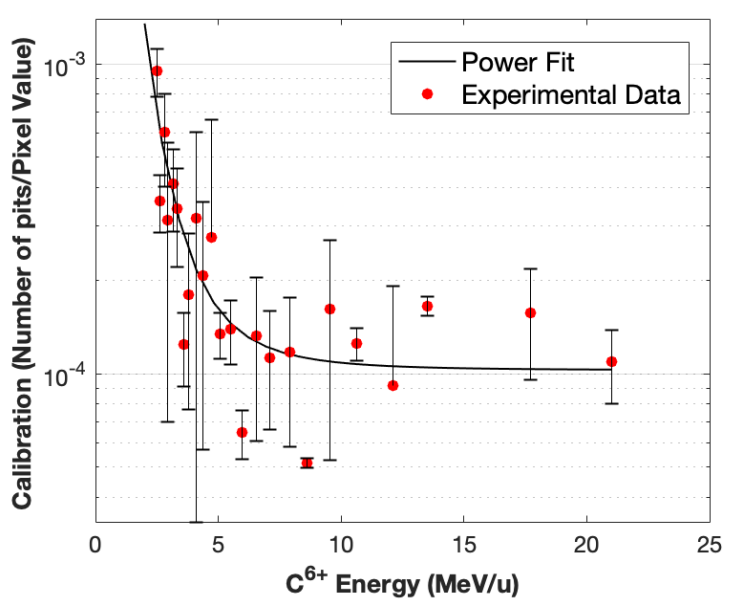

(a)

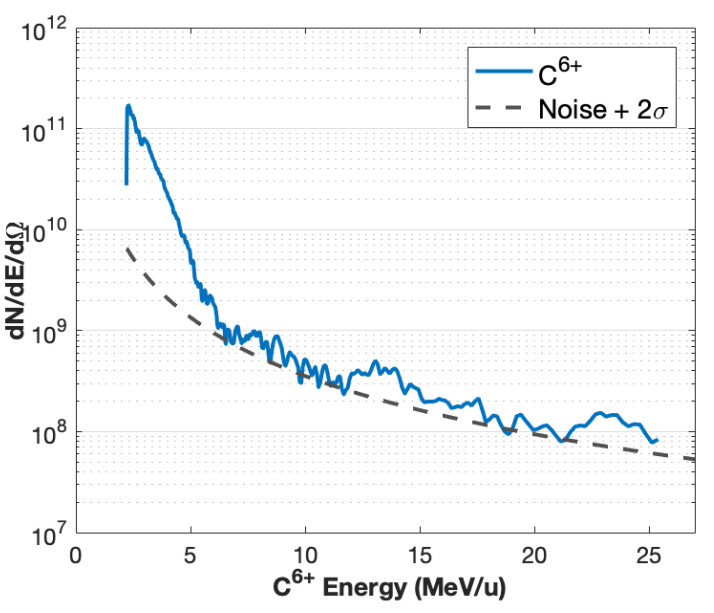

(b)

Figure 3: (a) Calibration function for each energy bin for TPS $0^{\circ}$ (red dots): Calibration $=$ $1.257 \times 10^{-2} \times$ energy $^{-3.331}+1.028 \times 10^{-4}$ (black line) (b) Spectrum for $15 \mathrm{~nm}$ amorphous carbon with intensity of $4.7 \times 10^{20} \mathrm{~W} / \mathrm{cm}^{2}$. The maximum energy is $25 \mathrm{MeV} / \mathrm{u}$

$[12,13]$, allows the direct comparison "in-situ" of the two detectors using the laser-accelerated ion signal.

As the carbon ions stop in the $1 \mathrm{~mm}$ thick CR-39, they could not reach the MCP. A segment of a parabolic trace will therefore be intercepted by the CR-39, resulting in 'shadows' on the MCP signal in correspondence to the CR-39 bars. A signal is produced on the MCP through the slotted gaps. At the interface between the MCP and CR-39, the CCD pixel value and CR-39 carbon pit density can be directly compared. The parabolic trace of carbon ions is now composed of alternating MCP and CR-39 segments at different energies so that a calibration curve can be created using the data at each interface (figure $2 \mathrm{~b}$ ). The CR-39 was processed after the experiment by etching it in a $6 \mathrm{M}$ solution of $\mathrm{NaOH}$ at $85^{\circ} \mathrm{C}$ in short intervals. Each bar was then imaged using a microscope and an image of the full ion trace could be built up. The pits could then be counted using the ImageJ software.

The ion trace on the detector has a finite width depending on the size of the pinhole, hence the total particle number at a given energy is the sum of the particle counts across this width. The total pixel value for a particular energy of the parabolic trace is accumulated across the width and compared to the sum of the carbon pits on the corresponding piece of CR-39 to relate the two totals. Since each pixel is equal to $100 \mu \mathrm{m}$, each pixel has an associated energy of carbon ion. This resolution was then applied to counting carbon pits in the CR-39, the total number of pits were counted in the first $100 \mu \mathrm{m}$ from the interface and accumulated. This was then plotted against the energy at the interface and a function produced as seen in figure $3 \mathrm{a}$ $\left(\right.$ Calibration $=1.257 \times 10^{-2} \times$ energ $\left.y^{-3.331}+1.028 \times 10^{-4}\right)$. The response can be seen to be mostly flat above $10 \mathrm{MeV} / \mathrm{u}$ which is promising for extending this to higher energies, as it indicates that, even if the particle's Bragg peak is not located within the confines of the MCP, it will still deposit a measurable amount of energy. 
The uncertainties associated with each energy bin arise from human error in counting of the pits per energy bin but also due to small scale spectral fluctuations which are detected only on the MCP or CR-39 but not the other. At these points, the MCP image and CR-39 interface would not correspond to the same number of particles and leads to the fluctuations of the calibration above and below the best fit line.

\subsection{Spectra}

The calibration can then be applied to the sum of the pixel value across the ion trace for every energy to produce an absolute number of particles. By knowing the solid angle of the entrance pinhole of the TPS, in this case $118 \mathrm{nSr}$, one can produce an absolutely calibrated spectrum in particle number per $\mathrm{MeV}$ per steradian. The noise of the detector is also shown in figure $3 \mathrm{~b}$ alongside a $\mathrm{C}^{6+}$ spectrum. The maximum ion energy is then determined to be at the point when the ion signal trace is equal to the noise $+2 \sigma$. The noise is measured as the signal of a parabolic trace on the detector alongside the carbon signal where there is no ion trace present. A moving average and standard deviation was calculated for this trace and plotted. As mentioned before, previous experiments [3] irradiated similar targets on Gemini but employed Image Plates in the TPS. Using MCPs and similar targets, we are able to quantitatively reproduce these spectra, which confirms the correctness of the calibration. In obtaining particle spectra as in figure $3 \mathrm{~b}$, the calibration was extended to energies higher than $21 \mathrm{MeV} / \mathrm{u}$ assuming the same power law.

\section{Conclusions}

The response of a Thomson Parabola Spectrometer - MCP assembly was calibrated for absolute particle number up to $21 \mathrm{MeV} / \mathrm{u}$. The response is best described using a power law. This has allowed for a calibrated spectrum to be produced for $\mathrm{C}^{6+}$ which is a very important measurement for bulk ion acceleration from thin foils. The experiment shows that MCPs provide a promising method of detecting high energy carbon ions and producing spectra from high repetition rate, high power laser systems. Data associated with the research published in this article can be accessed at: https://doi.org/10.17034/e5ed635c-0cc0-46af-aeea-782008172d6c

\section{Acknowledgments}

The authors acknowledge facility access provided by the Science and Technology Facility Council and grant support from the Engineering and Physical Sciences Research Council (grant. EP/K022415/1). The authors also acknowledge support from the laser and technical staff and target fabrication group at the Rutherford Appleton Laboratory.

\section{References}

[1] A. Macchi, M. Borghesi and M. Passoni, Ion acceleration by superintense laser-plasma interaction, Rev. Mod. Phys. 85 (2013) 751

[2] T. Esirkepov et al, Highly Efficient Relativistic-Ion Generation in the Laser-Piston Regime, Phys. Rev. Lett., 92 , (2004), 175003 
[3] C. Scullion et al, Polarization Dependence of Bulk Ion Acceleration from Ultrathin Foils Irradiated by High-Intensity Ultrashort Laser Pulses,Phys. Rev. Lett., 119, 054801

[4] S.V Bulanov et al, Oncological hadrontherapy with laser ion accelerators, Physics Letters A, 299, (2002), 240

[5] K. W. D. Ledingham et al, Towards Laser Driven Hadron Cancer Radiotherapy: A Review of Progress, Applied Sciences, 4, (2014) 402

[6] P. R. Bolton et al, Instrumentation for diagnostics and control of laser-accelerated proton (ion) beams, Physica Medica: European Journal of Medical Physics, 30, (2014), 255

[7] C. Scullion et al, Angularly resolved characterization of ion beams from laser-ultrathin foil interactions, Journal of Instrumentation, 11, (2016) C09020

[8] A. Mančić et al, Absolute calibration of photostimulable image plate detectors used as (0.5-20MeV) high-energy proton detectors, Review of Scientific Instruments, 79,(2008) 073301

[9] D. Doria et al, Calibration of BAS-TR image plate response to high energy (3-300 MeV) carbon ions, Review of Scientific Instruments, 86, (2015), 123302

[10] N. Rabhi et al, Calibration of imaging plate detectors to mono-energetic protons in the range 1-200 MeV, Review of Scientific Instruments, 86, (2017) 113301

[11] HamamatsuWebsite - ManualAccessed 01 - 02 - 2019https : //www.hamamatsu.com/jp/en/product/optical - sensors/electron_ion sensor/mcp/index.html

[12] Tae Won Jeong et al, Experimental evaluation of the response of micro-channel plate detector to ions with 10s of MeV energies, Review of Scientific Instruments, 87,(2016), 083301

[13] R. Prasad et al, Calibration of Thomson parabola - MCP assembly for multi-MeV ion spectroscopy,Review of Scientific Instruments, 623, (2010) 712 